\title{
Electronic and Thermoelectric Properties of $\mathrm{NiTiSi}$
}

\author{
S. Bati ${ }^{1,2}$, D. R. Jaishi ${ }^{1,2}$ and M. P. Ghimire ${ }^{1,2}$
}

${ }^{1}$ Central Department of Physics, Tribhuvan University, Kirtipur, Nepal

${ }^{2}$ Condensed Matter Physics Research Center, Butwal, Nepal
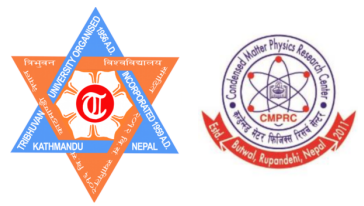


\section{Outline}

- Introduction

- Abstract

- Computational details

- Structure Optimization

- Results and discussions

- Electronic properties

- Thermolectric properties

- Conclusions 


\section{Abstract}

The electronic and thermoelectric properties of half-heusler compound NiTiSi has been studied using density functional theory and Boltzmann transport theory within the constant relaxation time approximation. NiTiSi is found to be an indirect band gap semiconductor with a band gap of $0.747 \mathrm{eV}$. Seebeck coefficient greater than 200 $\mu \mathrm{V} / \mathrm{K}$ at $1000 \mathrm{~K}$ is observed. The calculations suggests that p-type doping can significantly improve the thermoelectric properties of the compound with a maximum value of 0.13 at $1000 \mathrm{~K}$.

Keywords: Half-heuslers, Thermoelectrics, BoltzTraP 


\section{Introduction}

- Thermoelectrics can be used to generate electricity from waste heat.

- The efficiency of thermoelectric (TE) materials is measured by dimensionless figure of merit, ZT

$$
Z T=\frac{S^{2} \sigma T}{\kappa}
$$

where, $S$ is Seebeck coefficient, $\sigma$ is electrical conductivity,

$\mathrm{T}$ is the temperature and $\kappa$ is the total thermal conductivity of material

- A good TE material has ZT value greater than 1 .

- State of the art TE materials: PbTe, $\mathrm{Sb}_{2} \mathrm{Te}_{3}, \mathrm{Bi}_{2} \mathrm{Te}_{3}$
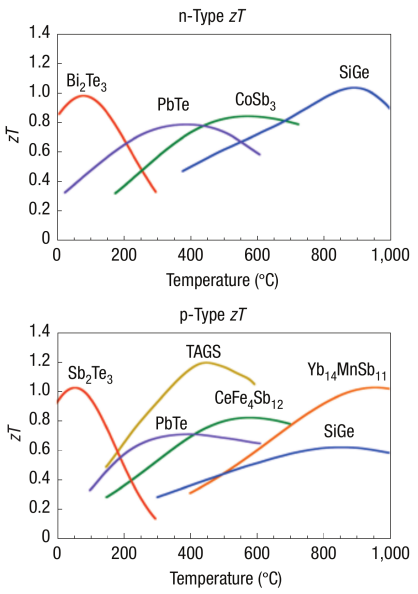

Figure: ZT values of some state of the art TE materials at different temperatures

(G. J. Snyder and E. S. Toberer, Nat. Mat. 7, 105 (2008) ) 


\section{Introduction:Half Heusler}

- TE materials have applications: power generation for space probes, temperature sensor etc.,

- Skuterrudites, chalcogenides, Heusler alloys, Clathrates commonly used TE materials.

- Half-heusler materials:

- Are mostly narrow band gap

- High temperature sustainable

- Generally non-toxic 


\section{Computational Details}

- Density functional theory calculations were performed using Full-potential linearized augmented plane wave + local orbital method as implemented in WIEN2k code.
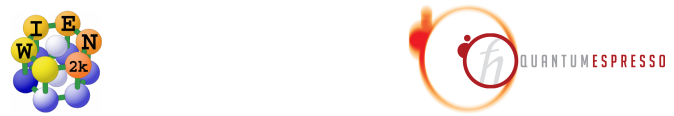

- $\mathrm{mBJ}$ exchange potential was used as it gives better estimate of band gap for semiconductors.

- BoltzTraP code was used to study transport properties and constant relaxation time approximation (CRTA) was used.

- Quantum Espresso package was used to calculate phonon dispersion. 


\section{Structure Optimization}

Space group F̄̄3m (216)

$\checkmark a=5.584 \AA, \alpha=90^{\circ}$

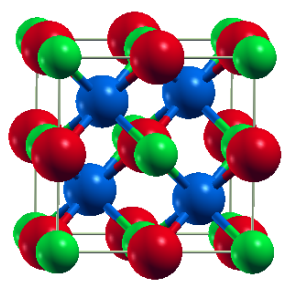

Figure: Conventional Unit cell of NiTiSi. Blue balls are $\mathrm{Ni}$, red are $\mathrm{Ti}$ and green balls are $\mathrm{Si}$.

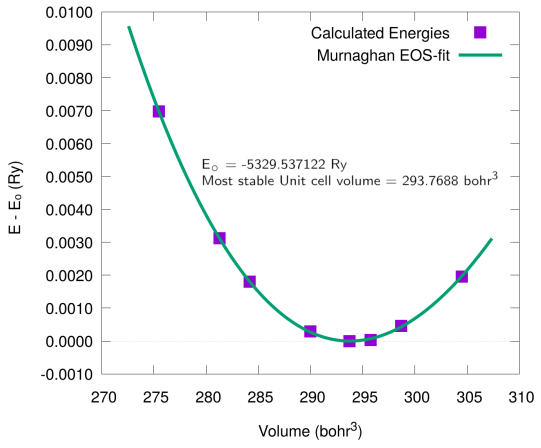

Figure: Volume optimization of NiTiSi. The displayed volumes are for primitive unit cell. $E_{\circ}$ is the energy for the most stable unit cell volume. 


\section{Results and Discussion}

Phonon Dispersion / Stability test

- The frequencies are real throughout the Brillouin zone.

- Thus, the crystal structure of NiTiSi is stable.

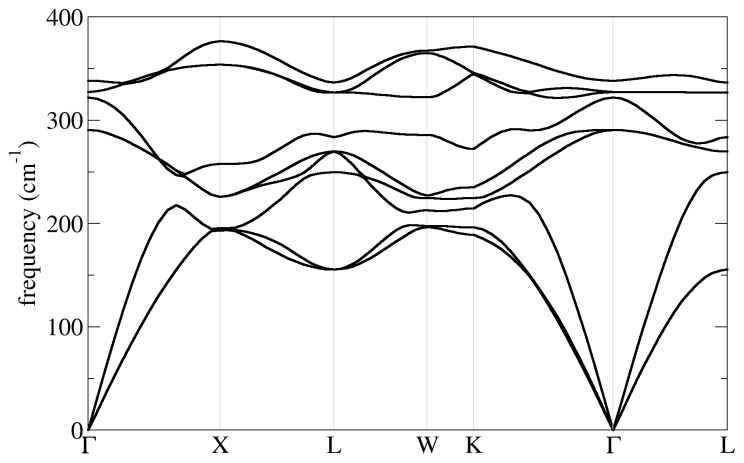

Figure: Phonon dispersion curves for NiTiSi 


\section{Results and Discussion}

Density of States

- Band gap of $0.759 \mathrm{eV}$ under GGA

- Band gap reduced slightly to $0.747 \mathrm{eV}$ under mBJ.

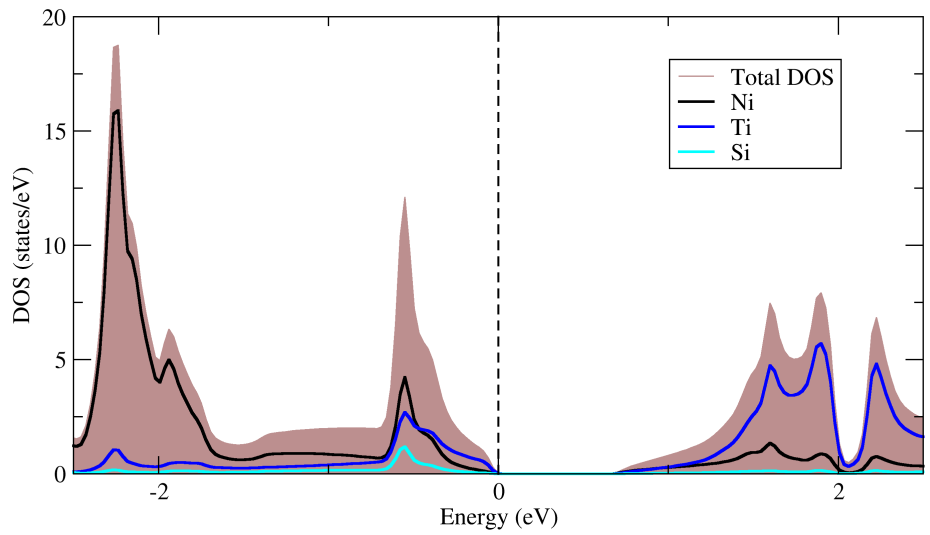

Figure: Total Density states for NiTiSi. 


\section{Results and Discussion}

\section{Density of States}

- Conduction region is dominated by Ti $3 d$ orbitals.

- Valence region is dominated by Ti-3d and Ni-3d orbitals.

- Hybridization of Ti-3d, Ni-3d and Si-3p states at $\sim-0.5 \mathrm{eV}$.

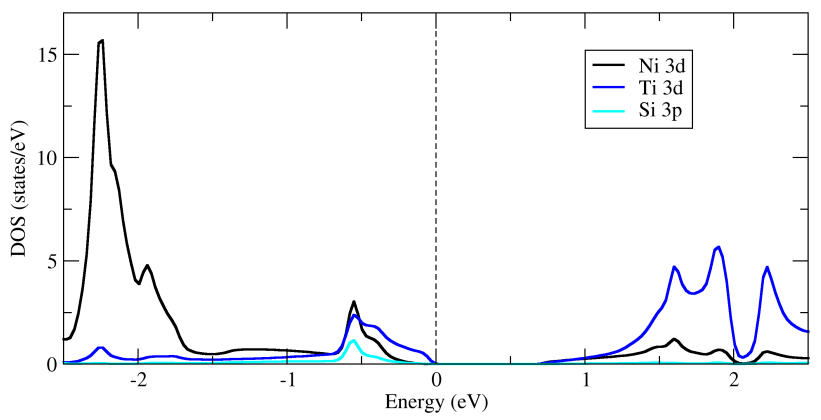

Figure: Partial Density of states Ni-3d, Ti-3d and Si-3p orbitals. This figure shows the contributions from orbitals with major contribution to DOS near Fermi level. 


\section{Results and Discussion}

- NiTiSi is an indirect gap semiconductor

- Band gap $=0.747 \mathrm{eV}$

- Valence band seems to be heavier than the conduction band.

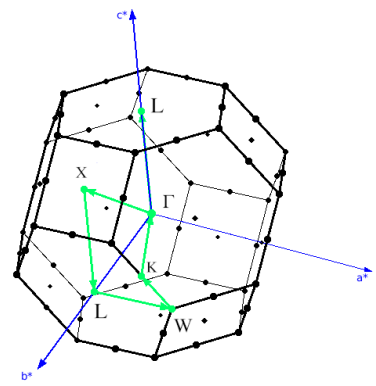

Figure: Selected K- path for band structure

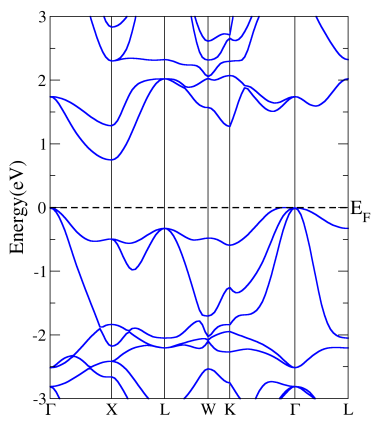

Figure: Band structure along high-symmetry point. 


\section{Results and Discussion}

\section{Transport Properties}

- In order to check the shift of Fermi level with temperature, effective mass of charge carriers were calculated.

- At $300 \mathrm{~K}$ the Fermi level is shifted by about $1.835 \mathrm{eV}$ towards conduction band.

High-symmetry point

Effective mass $\left(\mathrm{m}^{*} / \mathrm{m}_{e}\right)$

Valence Band $(\Gamma-\mathrm{K} \Gamma)^{\dagger}$

$\mathrm{HH} 1$

$\mathrm{HH} 2$

$-8.71$

LH

$-1.48$

$-0.45$

Conduction band (X-ГX)

Electron

3.38

Conduction band (X-XL)

Electron

0.91

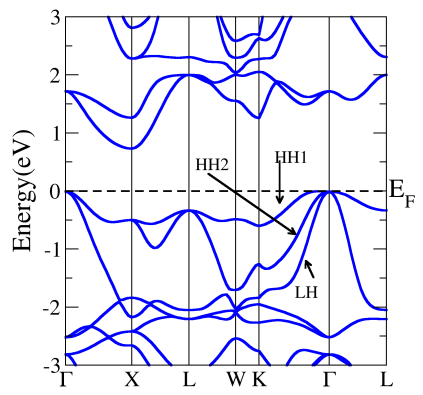

$\dagger$ The notation $\Gamma-K \Gamma$ denotes that the effective mass is calculated at $\Gamma$ point along $K \Gamma$ direction in primitive reciprocal lattice space and similarly for others too. 


\section{Results and Discussion}

\section{Transport Properties}

- At all temperatures Seebeck coefficient has positive values. So NiTiSi is expected to be a p-type semiconductor.

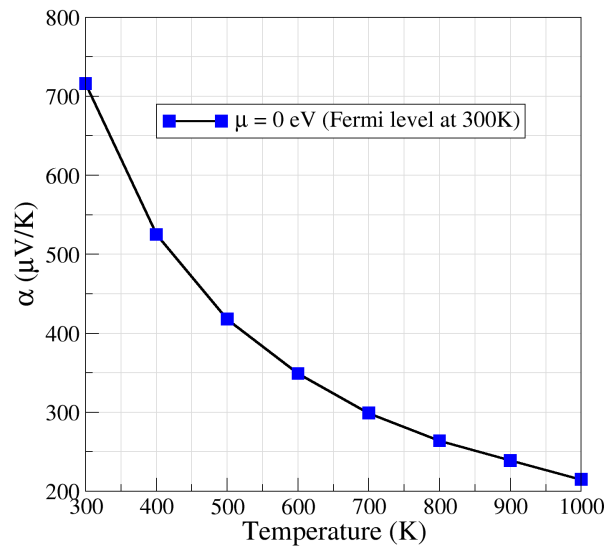

Figure: Seebeck coefficient vs Temperature plot for NiTiSi. 


\section{Results and Discussion}

- Power factor $(\mathrm{PF})=\mathrm{S}^{2} \sigma$, where $\mathrm{S}$ is Seebeck coefficient and $\sigma$ is the electrical conductivity.

- Power factor increases with temperature for both p-type and n-type doping.

- Larger values of maxima for negative $\mu$ implies that $p$-type doping will be more stable for enhancing the TE properties of NiTiSi.

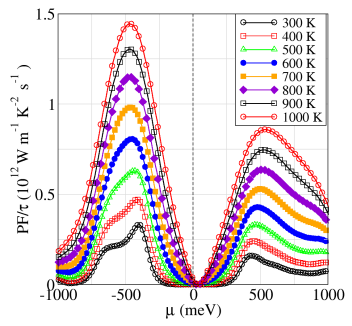

- Using a constant relaxation time for charge carriers, $\tau=2 \times 10^{-15} \mathrm{~s}$, the maximum value of $\mathrm{ZT}$ attainable by $p$-type doping is 0.01 at $300 \mathrm{~K}$ and rises upto 0.13 at $1000 \mathrm{~K}$.

Figure: Power factor vs chemical potential plot for NiTiSi. Power factor is coupled with the relaxation time $\tau$ 


\section{Conclusions}

- Electronic and TE properties of NiTiSi were studied using density functional theory.

- Band gap of NiTiSi was found to be $0.747 \mathrm{eV}$.

- Large value of Seebeck coefficient greater than $200 \mu \mathrm{V} / \mathrm{K}$ observed even at $1000 \mathrm{~K}$ signifies suitability of NiTiSi for high temperature TE applications.

- p-type doping is more favorable to enhance the ZT value of NiTiSi.

- ZT value can be increased upto 0.13 at $1000 \mathrm{~K}$. 
Thank you! 